\title{
Improving Transit Predictions of Known Exoplanets with TERMS
}

\author{
S.R. Kane ${ }^{1}$, D. Ciardi $^{1}$, D. Fischer ${ }^{2}$, G. Henry ${ }^{3}$, A. Howard ${ }^{4}$, E. Jensen ${ }^{5}$,
} G. Laughlin ${ }^{6}$, S. Mahadevan ${ }^{7}$, K. von Braun ${ }^{1}$, J. Wright ${ }^{7}$

${ }^{1}$ NASA Exoplanet Science Institute, Caltech, Pasadena, CA, 91125, USA

2 Dept of Astronomy, Yale University, New Haven, CT, 06520, USA

3 Tennessee State University, Nashville, TN, 37209, USA

${ }^{4}$ Dept of Astronomy, University of California, Berkeley, CA, 94720, USA

${ }^{5}$ Dept of Physics \&5 Astronomy, Swarthmore College, Swarthmore, PA, 19081, $U S A$

${ }^{6}$ UCO/Lick Observatory, University of California, Santa Cruz, CA, 95064, USA

7 Dept of Astronomy $\&$ Astrophysics, Pennsylvania State University, University Park, PA, 16802, USA

\begin{abstract}
.
Transiting planet discoveries have largely been restricted to the short-period or low-periastron distance regimes due to the bias inherent in the geometric transit probability. Through the refinement of planetary orbital parameters, and hence reducing the size of transit windows, long-period planets become feasible targets for photometric follow-up. Here we describe the TERMS project that is monitoring these host stars at predicted transit times.
\end{abstract}

\section{Introduction}

Monitoring known radial velocity (RV) planets at predicted transit times, particularly those planets in relatively eccentric orbits, presents an avenue through which to investigate the mass-radius relationship of exoplanets into unexplored regions of period/periastron space (Kane \& von Braun 2008, 2009). Here we describe techniques for refining ephemerides and performing follow-up observations (Kane et al. 2009). These methods are used by the Transit Ephemeris Refinement and Monitoring Survey (TERMS).

\section{Transit Ephemerides}

The transit window as described here is defined as a specific time period during which a complete transit (including ingress and egress) could occur for a specified planet. The size of a transit window will increase with time due to the uncertainties in the fit parameters, and thus follow-up of the transit window as soon as possible after discovery is optimal. Figure 1 (upper panel) shows the size of the transit window for a sample of 245 exoplanets. The transit windows of the short-period planets tend to be significantly smaller than those of long-period planets since, at the time of discovery, many more orbits have been monitored to provide a robust estimate of the orbital period. TERMS 


\section{EPJ Web of Conferences}
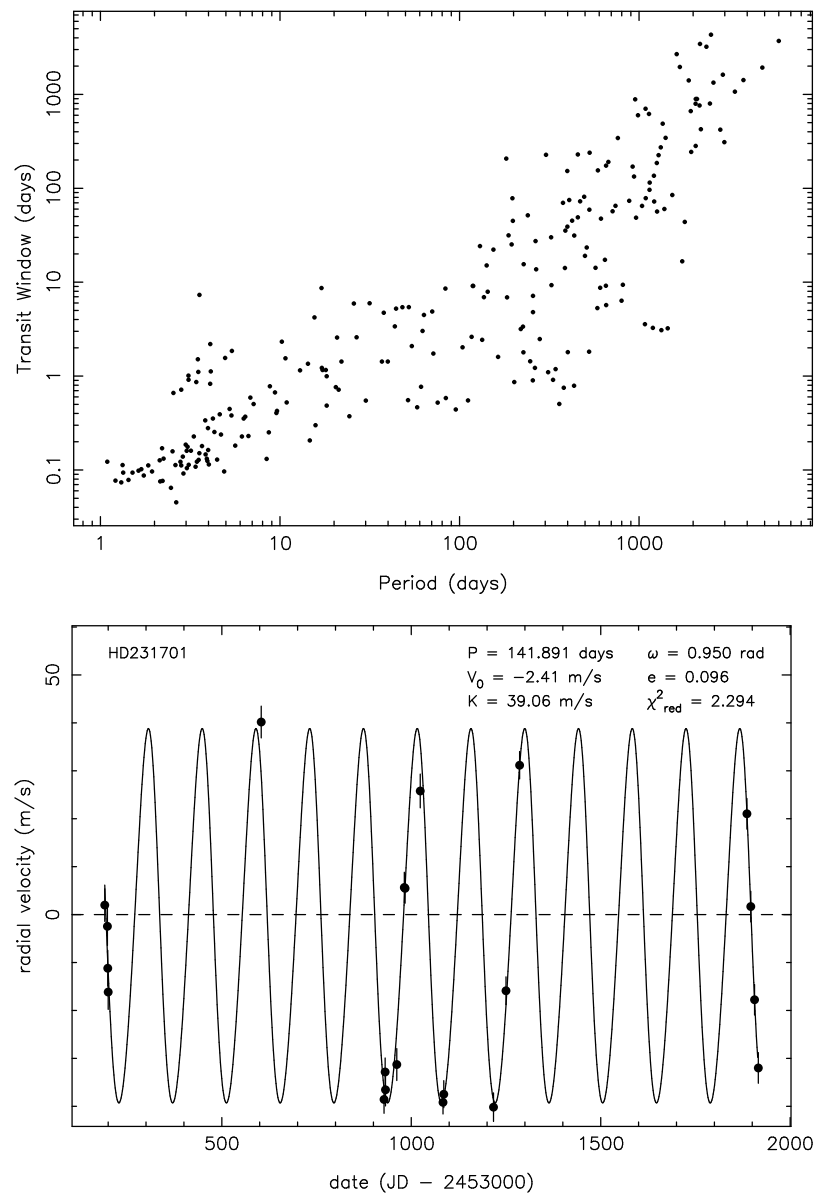

Figure 1: Upper panel: Calculated transit windows for a subset of the known exoplanets. Lower panel: Published RV data for HD $231701 b$ with four additional simulated measurements.

chooses targets that have small transit windows, medium-long periods, and a relatively high probability of transiting the host star.

\section{Refining the Ephemerides}

The transit ephemeris for a particular planet can often be significantly improved with the addition of a handful of high-precision RV data. For example, the planet orbiting the star HD 231701 (Fischer et al. 2007) has a current transit window of $\sim 82$ days based upon the discovery data. The addition of four subsequent measurements as shown in Figure 1 (lower panel) would improve both the precision of the period and time of periastron passage, resulting in a reduction of the transit window to 3.7 days - a factor of almost 25! Through selective observations at optimal times, we produce viable targets for photometric follow-up. 


\section{TERMS Results}

A considerable number of high transit probability targets are difficult to monitor adequately during their transit windows because the uncertainties in the predicted transit mid-points are too high. The acquisition of a handful of new RV measurements at carefully optimised times can reduce the size of a transit window by an order of magnitude. This is described in more detail by Kane et al. (2009).

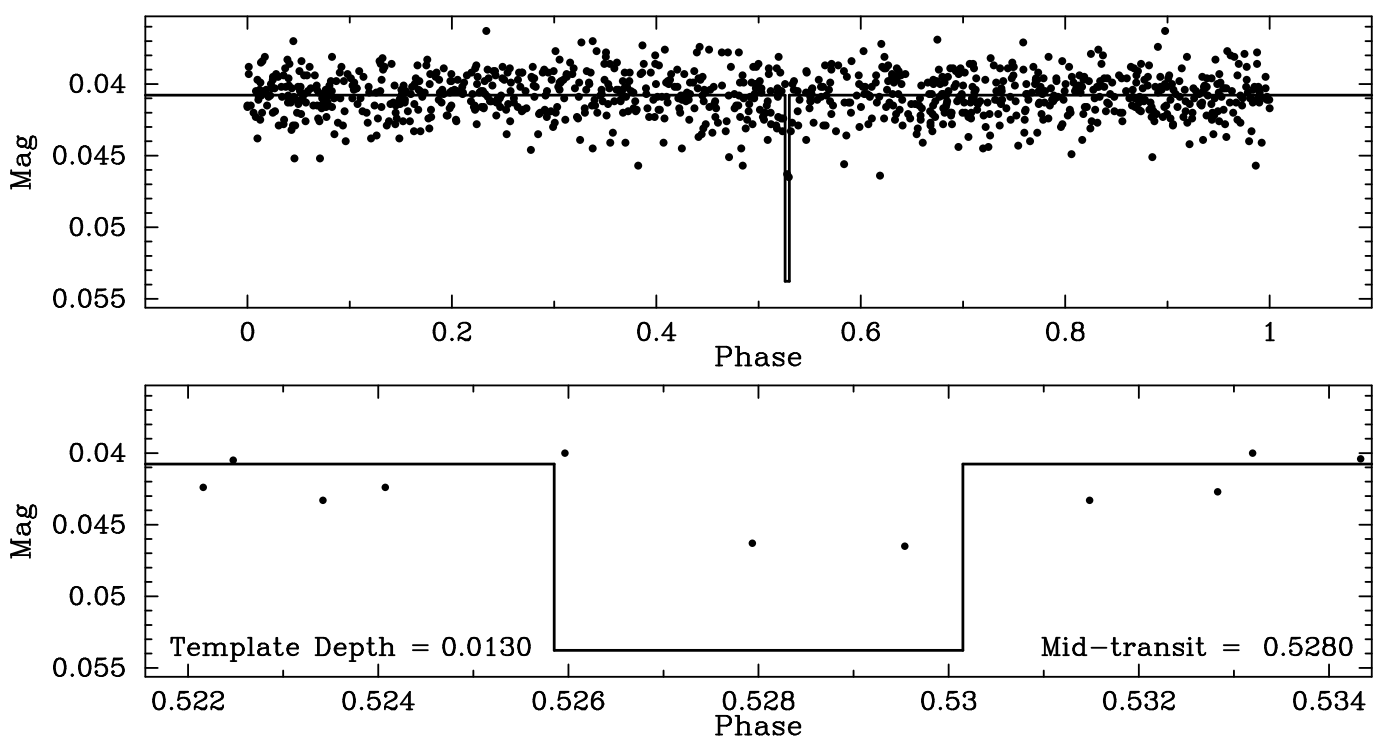

Figure 2: Two panels showing the ruling out of a transit by the planet orbiting HD 114762 using data from the T10 APT.

Figure 2 presents data acquired with the T10 0.8m Automated Photoelectric Telescope (APT) during a predicted transit time of HD 114762b. The transit window was refined to less than a day using Lick RV data, and the transit of this planet was subsequently ruled out. The observations from this survey will lead to improved exoplanet orbital parameters and ephemerides even without an eventual transit detection. The results from TERMS will provide a complementary dataset to the fainter magnitude range of the Kepler mission, expected to discover many intermediate to long-period transiting planets.

\section{References}

Fischer, D.A., et al. 2007, ApJ, 669, 1336

Kane, S.R., \& von Braun, K. 2008, ApJ, 689, 492

Kane, S.R., \& von Braun, K. 2009, PASP, 121, 1096

Kane, S.R., et al. 2009, PASP, 121, 1386 